\title{
Lattice Boltzmann and
} \section{nonextensive diffusion}

\author{
Bruce M. Boghosian ${ }^{1}$ and Jean Pierre Boon ${ }^{2 *}$ \\ ${ }^{1}$ Mathematics Department, Tufts University, Medford, MA 02155 \\ ${ }^{2}$ Physics Department, Université Libre de Bruxelles, B-1050 \\ Bruxelles, Belgium \\ *bruce.boghosian@tufts.edu,jpboon@ulb.ac.be
}

\begin{abstract}
tatistical physics today is arguably in much the same situation $\checkmark$ that Euclidean geometry found itself in the early nineteenth century. Over the last decade, an increasing body of evidence has indicated that denying a certain postulate of statistical physics the extensivity of the entropy - results not in a contradiction, but rather in an entirely new family of mathematically consistent variants of the statistical physics developed by Boltzmann and Gibbs (see Editorial).

The mathematical formulation of these variants begins with a generalization of the definition of the entropy in terms of the microscopic state probabilities of the system under study (see Box 1 in the Editorial). A family of such entropies has been posited, parametrized by a single positive number $q$, such that the usual Boltzmann- Gibbs formulation is recovered when $q=1$. More precisely, whereas the Boltzmann-Gibbs entropy is expressed in terms of the logarithm function, nonextensive variants are expressed in terms of a q-deformed logarithm (defined in Box 1) to which application of l'Hôpital's rule confirms reduction to the ordinary logarithm as $q \rightarrow 1$. Remarkably, many fundamental results of statistical physics, such as the Maxwell relations and Onsager reciprocity, are " $q$-invariant"; that is, they hold for any statistical physics in the family. Other results, such as the Fluctuation-Dissipation Theorem and the compressible Navier-Stokes equations for viscous fluid dynamics, must be modified by the addition of terms that vanish when $q=1$.
\end{abstract}

\section{Entropic Lattice Boltzmann Models}

In the late 1980's and early 1990's, it was realized that lattice kinetic models could be used to great advantage in the construction of new algorithms for computational fluid dynamics [1]. These are models wherein particles hop about on a regular spatial lattice in discrete time steps according to deterministic rules, with velocities restricted to the lattice vectors, and with collisions conserving mass and momentum. Unlike particles in a continuum fluid, whose velocities take on values in $\mathbb{R}^{3}$, the velocity space of a lattice kinetic model consists of a finite set of points. Hydrodynamic quantities - such as mass density and momentum density - may be obtained from such a discrete-velocity distribution by a finite sum, rather than an integral.

It is possible to construct Boltzmann equations for the singleparticle distribution function for lattice fluids. At first, researchers restricted their attention to Boltzmann equations for microscopic models of discrete-velocity particles, but it was then realized that lattice Boltzmann equations for discrete-velocity fluids could be constructed with idealized collision operators that did not correspond to any underlying particulate model [2]; even a single relaxation time operator - à la Bhatnagar-Gross-Krook (BGK) replacing the full collision operator, could be used for this purpose [3] giving rise to so-called lattice BGK equation: $f_{j}\left(r+v_{j} \Delta t, t+\Delta t\right)$

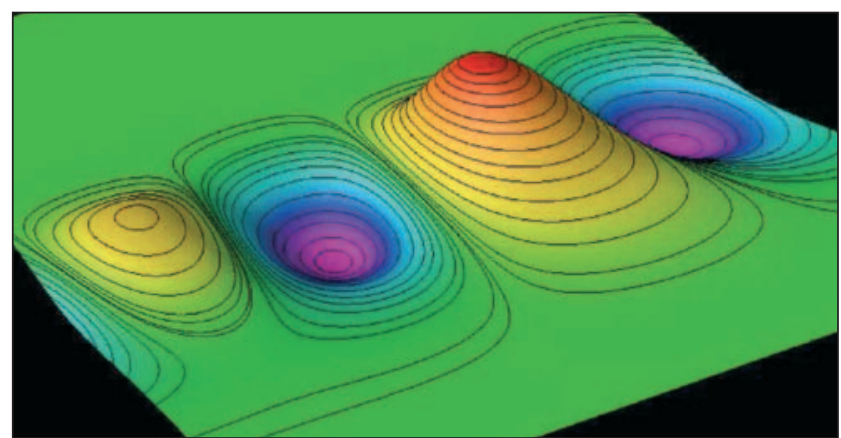

$\Delta$ Fig. 1: Concentration fluctuations field in the onset of fingering between two miscible fluids (flow direction SouthEast) showing landscape of q-Gaussian "hills and wells" (color code indicating highest positive values (red) to largest negative values (magenta) of c). These structures identify the existence of precursors to the fingering phenomenon as they develop before any fingering pattern can be seen.

- $f_{j}(r, t)=\tau^{-1}\left(f_{j}(r, t)-f^{\text {equil }}\right)$, where $f_{j}$ is the single-particle distribution function for velocity $v_{j}$, position $r$, and time $t$.

Lattice Boltzmann equations derived from underlying microscopic dynamical models with detailed balance possess a discrete-space-time analog of Boltzmann's celebrated $H$-Theorem, which establishes that when the system evolves towards equilibrium it will reach a state of maximum entropy. That is - in mathematical terms - it is possible to identify a Lyapunov function for the dynamics. From the point of view of the lattice Boltzmann equation as a physical model, this maintains an important property possessed by the continuum Boltzmann equation. However lattice BGK models are not based on any underlying microscopic dynamical model, and one of the properties lost in the transition from lattice models with a microscopic basis to lattice models without one was the $H$-Theorem. The effort to modify the lattice BGK model so that it has an $H$-Theorem led to the proposal of so-called entropic lattice Boltzmann models in the late 1990's. These models begin by positing an $H$ function of trace form, depending on the single-particle distribution function, and by dynamically adjusting the relaxation time in the BGK operator to ensure that this $H$ function does not increase.

Researchers then focussed efforts on finding the most general class of entropic lattice Boltzmann model that would produce correct macroscopic behavior, i.e. give rise to the Navier-Stokes equations in the hydrodynamic limit [4]. These analyses restrict attention to collision operators of BGK form, but with variable relaxation time. They do not specify the precise form of the $H$ function; rather, they assume that $H$ is of trace form $H(t)=\sum_{\mathbf{r}} \sum_{j} h\left(f_{j}(\mathbf{r}, t)\right)$, where the outer sum is over the lattice and the inner sum is over the finite set of velocities, without specifying the form of the function $h$. Boltzmann's $H$ function would correspond to the choice $h(f)=f \ln f$, but that assumption is not made here. Of course the form of the equilibrium distribution function will depend on the choice of $h$. Remarkably, it is possible to carry out the entire Chapman-Enskog analysis for the above-described model without ever specifying $h$. This results in the Navier-Stokes equations with an extra factor multiplying the advective term, $\mathbf{v} \cdot \boldsymbol{\nabla}$ v. This extra factor depends on $\mathrm{h}$ and its first two derivatives. The Galilean invariance of the Navier-Stokes equations depends on there being no such extra factor multiplying the advective term. The convective derivative operator is Galilean invariant. The above-described extra factor breaks that Galilean invariance. The 
breaking of Galilean invariance is not completely unexpected after all, the lattice itself constitutes a preferred Galilean reference frame, and this problem had been noted in earlier microscopic particulate lattice models of hydrodynamics. But now, however, it is straightforward to restore Galilean invariance; we need only demand that this extra factor be equal to one. Since the extra factor depends on $h$ and its first two derivatives, this results in a second-order nonlinear ordinary differential equation for $h$, whose solution is $h(f)=f \ln _{q} f$, where $\ln _{q}$ is precisely the $q$-logarithm function [5] used in nonextensive statistical physics!

What this analysis shows is that lattice hydrodynamics provides an example of a statistical mechanical system from which the $q$ deformed functional form arises naturally, and can be demonstrated from first principles. It should be emphasized that Boltzmann's $H$ function is not the entropy! The entropy is a functional of the $N$ body distribution function, whereas Boltzmann's $H$ function is a functional of the single-particle distribution function. Nevertheless, it is striking that just as the natural logarithm function appears in Boltzmann's entropy (see Box 1 ) and in Boltzmann's $H$ function, the $q$-deformed logarithm function appears in the nonextensive entropy, and in the $H$ function for an entropic lattice Boltzmann model. It is however important to acknowledge that an entropic lattice Boltzmann model is not a natural physical system, but a highly idealized model system (that has found importance in numerical analysis). The analysis described above suggests that either the fragmentation of space into a regular lattice, or the reduction of velocity space to a finite set of points, or perhaps both, has resulted in the natural appearance of the $q$-deformed logarithm. It may be that similar fragmentation of phase space, possibly due to loss of ergodicity - wherein time averages are not equal to phase space averages - gives rise to the appearance of this same functional form in the entropy of certain complex systems.

\section{Nonextensive diffusion as nonlinear response}

One of the characteristic features of nonextensive statistical mechanics is the appearance of non-exponential distribution functions with power-law tails and there has been considerable interest in the question of how such non-exponential distributions might arise from first principle consideration [6]. For the diffusion processes, power-law distributions follow, in a generic manner, from a generalization of classical statistical mechanics linear response theory. Suppose we are interested in the diffusion of a tracer particle in a fluid. We then consider the probability $f\left(\mathbf{r}, t ; \mathbf{r}_{0}, 0\right)$ to find the diffuser at point $\mathbf{r}$ at time $t$ given that it starts at point $r_{0}$ at time 0 . This distribution function - but, instead of probabilities, we could as well speak of the concentration of a diffusing species (as in the example below) - as we know from linear response theory, obeys the advection-diffusion equation.

Now if one makes the key assumption that the current is not simply described by gradients in the distribution function, but rather by gradients in the distribution raised to some power, one can proceed essentially along the same lines of reasoning as in classical response theory, and obtain a generalized diffusion equation

$$
\begin{aligned}
\frac{\partial}{\partial t} f\left(\mathbf{r}, t ; \mathbf{r}_{0}, 0\right) & =\frac{\partial}{\partial \mathbf{r}} \cdot \mathbf{v}(\mathbf{r}, t) f\left(\mathbf{r}, t ; \mathbf{r}_{0}, 0\right) \\
& +\frac{\partial}{\partial \mathbf{r}} \cdot \mathbf{D}(t) \cdot \frac{\partial}{\partial \mathbf{r}} f^{\alpha}\left(\mathbf{r}, t ; \mathbf{r}_{0}, 0\right) .
\end{aligned}
$$

What makes this equation a generalized diffusion equation is the power $\alpha \neq 1$, and the generalized Einstein relation connecting the diffusion coefficient $D$ to the underlying microscopic dynamics and corresponding distribution function.
The generalized equation can be viewed as a classical diffusion equation with an effective diffusion coefficient $\mathrm{D}_{\alpha}=\alpha \mathrm{D} f^{\alpha-1}$. Since it is quite common to introduce concentration-dependent and density-dependent diffusion coefficient to describe complex systems, this does not differ radically from classical phenomenology: what is unusual is that the effective diffusion coefficient vanishes when the probability (or concentration) vanishes. The generalized diffusion equation has formally the same structure as the "porous media equation", but the diffusion coefficient depends on the solution of the equation. This leads to the fact that the diffusion process is classical in the sense that the mean-squared displacement increases linearly with time, but the solutions are not Gaussian: they have the canonical $q$-exponential form (see Box 1) with $q+\alpha=2$.

\section{Precursor statistics}

From a pragmatic viewpoint, the lattice Boltzmann equation - in particular with the lattice BGK formulation (described above) can be used to simulate nonequilibrium systems [2]. In contrast to other computational methods, the lattice Boltzmann method offers a mesoscopic approach, which is based on a kinetic theoretical analysis where the macroscopic description (such as given e.g. by the set of hydrodynamic equations) is not pre-established. This approach was used for instance to investigate precursor phenomena in the onset of fingering and the data were analyzed using the generalized diffusion equation [8].

Fingering is a generic phenomenon that results from the destabilization of the interface between two fluids with different

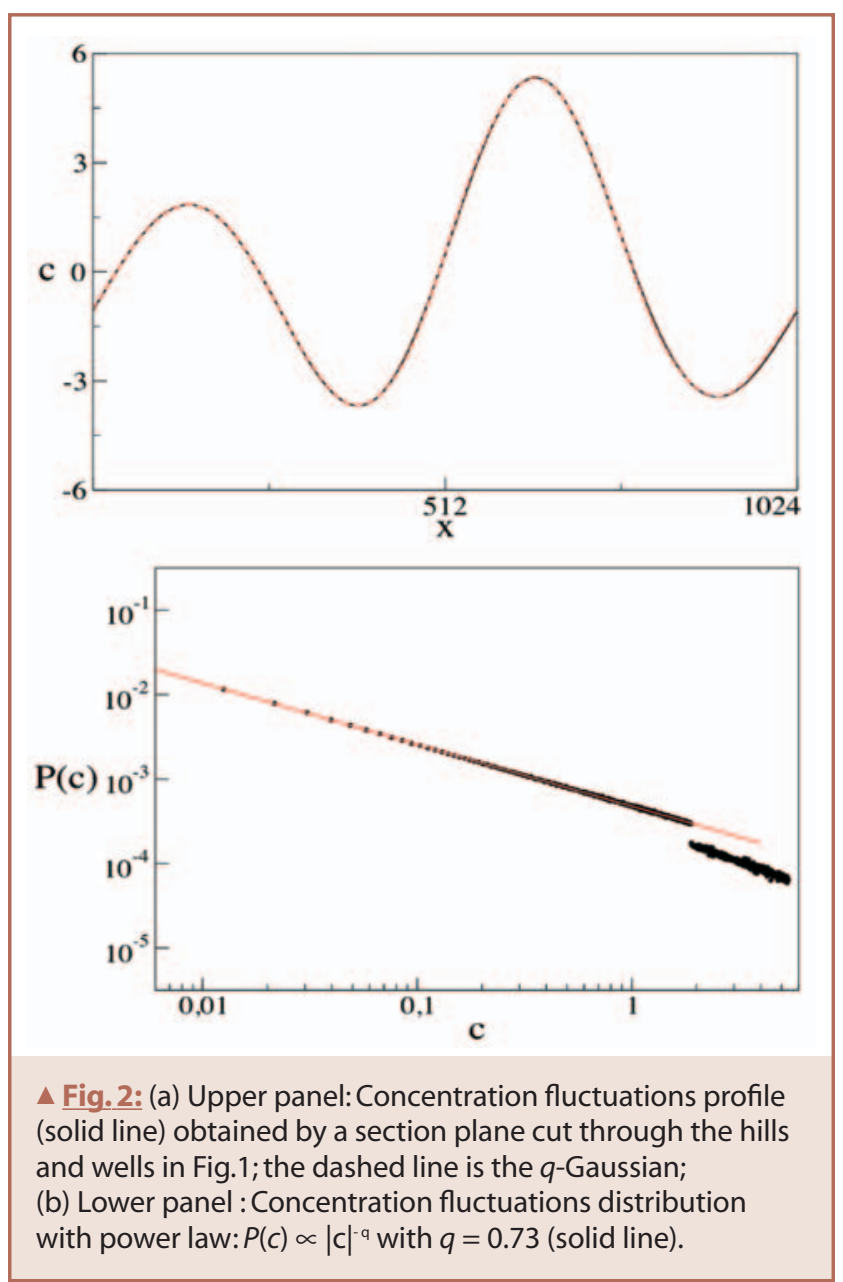


mobilities (because of their differences in viscosity or density) in systems such as a shallow layer or a porous medium, when the fluid with highest mobility is forced through the other fluid. As soon the system responds non-linearly to the driving force, enhanced internal fluctuations (such as concentration fluctuations) are produced characterizing the early stage of the fingering process. If the fluids are miscible, the mixing zone at the interface between the two fluids grows as the fluid with high mobility displaces the other fluid, and there is a dynamical transition where the exponent of the growth of the mixing length of the interfacial zone, $L_{\text {mix }} \propto t^{\mu}$, changes from $\mu=1 / 2$ (the value typical of a diffusive process) to a larger value. In the diffusive regime (before any fingering pattern becomes visible), the flow produces local concentration gradients which induce mobility fluctuations thereby triggering vorticity fluctuations. The concentration field in Fig.1 shows that a "landscape" of alternating hills and wells has developed. In each "blob", the concentration field exhibits a two-dimensional $q$-Gaussian profile as illustrated in the upper panel of Fig.2 obtained by a section plane cut through the extrema in Fig.1. Such $q$-Gaussians are precisely solutions to the generalized diffusion equation. Now the remarkable fact is that the distribution that follows from a $q$ exponential profile has a power law behavior. In two dimensions and for a $q$-Gaussian - as for the case of the concentration fluctuations $c$ in the fingering pre-transitional regime - the distribution is simply $P(c) \propto c^{-q}$, as illustrated in Fig. 2 .

The example presented here is representative of a generic class of driven nonequilibrium systems where $q$-exponentials and power law distributions are the signature of long-range interactions and whose dynamical behavior is governed by non-linear equations, such as the generalized equation described above.

What has been shown is that during the onset of fingering, one can identify precursors which exhibit statistical features typical of nonextensive statistics. Then the question arises as to whether there is a possible physical interpretation of the origin of nonextensivity? The driving force produces a spatial sequence of alternating structures, which, if they were independent, would exhibit an ordinary Gaussian profile originating from local diffusion centers ( $\delta$-functions), and would be described by a classical advection-diffusion formulation. However, when growing, these structures develop into overlapping Gaussian blobs, and what the analysis shows is that by renormalizing the overlapping Gaussians, they are recast into a sum of scale invariant independent $q$-Gaussians. Similar statistical properties have been obtained in other nonequilibrium systems which are discussed in companion articles in the present issue.

\section{References}

[1] J.-P. Rivet and J.P. Boon, Lattice Gas Hydrodynamics (Cambridge University Press, Cambridge, 2001).

[2] S. Succi, The Lattice Boltzmann Equation for Fluid Dynamics and Beyond (Clarendon Press, Oxford, 2001).

[3] Y. Qian, D. d'Humières and P. Lallemand, Europhysics Letters, 17, 479 (1992).

[4] B.M. Boghosian, P.J. Love, P.V. Coveney, S. Succi, I.V. Karlin and J. Yepez, Phys. Rev. E, 68, R025103 (2003).

[5] The precise value of $q$ is the solution of a transcendental equation with parameters depending on the spatial dimension, and the set of particle masses and velocities used.

[6] C. Tsallis Physica D, 193, 3 (2004).

[7] J.F. Lutsko and J.P. Boon, EuroPhys. Lett., 71, 906 (2005).

[8] P. Grosfils and J.P. Boon, Physica A, 358 (2005).

\section{Relaxation and aging in} a long-range interacting system

Francisco A. Tamarit ${ }^{1}$ and Celia Anteneodo ${ }^{2 *}$ ${ }^{1}$ Facultad de Matemática, Astronomía y Física, Universidad Nacional de Córdoba, Ciudad Universitaria, 5000 Córdoba, Argentina

${ }^{2}$ Departamento de Física, Pontificia Universidade Católica do Rio de Janeiro, CP 38017, 22452-970, Rio de Janeiro, Brazil

*tamarit@famaf.unc.edu.ar; celia@cbpf.br

C omplexity refers to the quality that certain systems possess of being intricate and hardly predictable. Ranging from the turbulent flows that form our atmosphere to the human languages, our life has plenty of examples of natural complex behavior. Statistical Mechanics, the area of physics that deals with the problem of explaining the macroscopic world from the dynamics of its components, faces nowadays the challenge of applying the standard reductionist program to all these fascinating systems.

Even when the mathematical equations for describing its time evolution may be only a few, a complex system is composed of a huge number of interacting constituents. These constituents, usually very simple ones, interact giving rise to the emergence of an unexpected collective phenomenology, where cause and effect become subtle and where the long time behaviour is no longer obvious. For succeeding in the plan of explaining complex behavior from first principles, physicists have been looking for simplified models, mathematically tractable and able to catch the essence of complexity.

Suppose you have such a complete knowledge on the microscopic details of certain system that you can write down its Hamiltonian. Now the natural question that arises is the following one: which are the mechanical conditions the system must fulfill in order to guarantee that the statistical mechanics calculations would predict, with an adequate degree of accuracy, the time averaged quantities obtained from a laboratory experiment. And when trying to answer such an apparently simple question, one discovers that even the simplest systems can give place to very intricate behaviour.

Perhaps the simplest Hamiltonian model of interacting particles one can image is the so called Hamiltonian Mean Field (HMF) model. Unlike most of the models we are used to deal with when modeling complexity, in this case, not only the dynamical variables but also the interactions among them are extremely simple, lacking any trait of randomness or frustration. The system consists of a set of $N$ interacting particles or rotators of unitary mass, each one confined to move around its own unitary circle [1]. Each particle is then mechanically described by an angle $\theta_{i}$ and the corresponding conjugate momentum $p_{i}$. The dynamics of the system is ruled by the following Hamiltonian:

$$
\mathcal{H}=\frac{1}{2} \sum_{i} p_{i}^{2}+\frac{1}{2 N} \sum_{i, j}\left[1-\cos \left(\theta_{i}-\theta_{j}\right)\right] \equiv K+V .
$$

The first term is the kinetic energy associated with the motion of the particles, while the second one corresponds to the interaction potential (the summation running over all different pairs of particles). 